\title{
The Suicide Prevention, Depression Awareness, and Clinical Engagement Program for Faculty and Residents at the University of California, Davis Health
}

\author{
Andrés F. Sciolla ${ }^{1}$ (D) Jessica Haskins ${ }^{1} \cdot$ Celia H. Chang ${ }^{1} \cdot$ Carol Kirshnit $^{1} \cdot$ Margaret Rea $^{1} \cdot$ Jeffrey Uppington ${ }^{1}$. \\ Peter Yellowlees ${ }^{1}$
}

Received: 25 October 2019 / Accepted: 15 March 2021/Published online: 2 April 2021

(C) Academic Psychiatry 2021

\begin{abstract}
Objective The objective of the study is to present results of a depression and suicide screening and treatment referral program for physicians at an academic medical center.

Methods An anonymous web-based screening questionnaire was sent to all physicians at a large academic center. Responses were classified as indicating either high, moderate, or low risk for depression and suicide. Physicians at high and moderate risk were contacted by a counselor through a messaging system. The counselor's message contained information on risk level and an invitation to meet in person. High-risk respondents who did not reply to the message or declined to meet received mental health resources. Respondents who met with the counselor were offered individualized treatment referrals and to participate in a 1-year follow-up of self-reports every 3 months.

Results The questionnaire was sent to approximately 1800 residents, fellows, and faculty from February 2013 through March 2019. A total of 639 questionnaires were received, 100 were excluded for various reasons, and 539 were used to conduct analyses (14.4\% response rate). The majority of respondents were classified at moderate (333 [62\%]) or high (193 [36\%]) risk for depression or suicide. Eighty-three respondents were referred for mental health care, and 14 provided data for the follow-up study.

Conclusions Results of screening physicians for depression and suicide at one academic medical center highlight the challenges of engaging most of them in this activity and the satisfaction of the minority who successfully engaged in a treatment referral program.
\end{abstract}

Keywords Depression screening $\cdot$ Physician burnout $\cdot$ Physician help-seeking $\cdot$ Physician well-being $\cdot$ Suicide screening

Traditional medical culture has emphasized the care of others over the care of self and afforded a low priority to physician mental health [1-3]. Physicians are notoriously reluctant to report their own health problems and seek professional help [4]. Many physicians do not regularly see a primary care physician [5] and are less likely than nonphysicians to follow health maintenance guidelines regarding annual checkup, colonoscopy, and mammogram [6]. A review of empirical studies found that self-diagnosis and self-treatment are prevalent among medical students and physicians [7]. These behaviors are embedded in the medical culture, which encourages self-reliance and the denial of

Andrés F. Sciolla

afsciolla@ucdavis.edu

University of California, Davis, Sacramento, CA, USA vulnerability $[8,9]$ and are learned implicitly during training, at least partly [10-13]. Physicians also delay care because of the perceived stigma associated with mental illness and concern at the possible professional consequences of revealing a mental disorder $[14,15]$. These barriers to help-seeking may heighten the mental health crisis among physicians and medical trainees as a result of the coronavirus disease 2019 (COVID-19) pandemic. A surge in COVID-19-related stressors and related distress has been documented worldwide in this population [16-20].

Age-standardized suicide mortality ratios (i.e., populationbased) are significantly higher in female physicians compared with women in general and are significantly lower in male physicians compared with men in general [21]. Although suicide rates among residents as a group are lower than rates in the general US population, suicide is the leading cause of death in male residents and the second-most-prevalent for female residents [22]. 
The role of the multidisciplinary University of California, Davis Health (UCDH) Well-Being Committee (WBC) is to provide assistance to impaired medical staff members, as described elsewhere [23]. In 2011, the WBC started a program for suicide screening, assessment, and referral of house staff and faculty similar to the one at the University of California, San Diego School of Medicine (UCSD) [24]. Like the UCSD program, ours used a web-based interactive screening program developed by the American Foundation for Suicide Prevention $[25,26]$.

The purpose of this study is to present a summary of results from five survey waves of the UCDH Suicide Prevention, Depression Awareness, and Clinical Engagement Program over 6 years. Specifically, we sought (1) to examine the effectiveness of an anonymous online screening and confidential referral process for medical staff who need mental health and/ or treatment and (2) to assess the effectiveness of treatment referrals at 3, 6, 9, and 12 months after an in-person evaluation of physicians who agreed to break their anonymity.

\section{Method}

The study's methodology has been described previously, along the program's first year of results. The UCDH Suicide Prevention, Depression Awareness, and Clinical Engagement Program is funded internally by UCDH and is managed by the WBC. The primary counselor secured for the project is a psychologist employed within the Employee Assistance Program. The online screening tool was purchased from the American Foundation for Suicide Prevention and white-branded as the UCDH Wellness Survey. The survey was accessed by respondents through a link included in an email invitation sent by the chair to residents and faculty of each department or, in the past two years, by the Chief Wellness Officer. The invitation emphasizes confidentiality and IRB approval. The survey can also be accessed directly through the WBC's website, which contains a description of the screening program, information about confidentiality, emergency contact information, and a list of therapists and psychiatrists who agreed to provide care to UCDH residents (this group includes fellows pursuing subspecialty training) and faculty. Respondents were asked to provide a professional identity (i.e., medical student, resident or fellow, physician faculty, non-physician faculty, nurse or nurse practitioner, and prefer not to answer). No other demographic data were collected.

\section{Data Collection and Analysis}

The main goal of the UCDH Suicide Prevention, Depression Awareness, and Clinical Engagement Program is to save lives and ameliorate suffering by providing feedback on risk level and referrals for treatment to as many unique physicians as possible. Therefore, we send the survey to all UCDH faculty and residents, every year. Since most of them work or train at UCDH for several years, we anticipated that most would receive the survey multiple times. This posed a challenge to data collection and analysis. For example, if the same 20 physicians responded every year, we could not say that we gathered data on 40 physicians in 2 years. It would still be the same 20 physicians being screened and referred to treatment. We addressed this challenge in the numerator by removing duplicate responses, i.e., if the same email is used to respond more than once. We addressed this challenge in the denominator by only adding new respondents to the baseline cohort to obtain a net total of unique respondents. Otherwise, respondents would have been counted in the denominator multiple times.

\section{Screening Tool}

The Wellness Survey is a confidential online assessment of stress, depression, and other mental health concerns aimed at identifying depression and suicide risk and facilitating access to mental health services $[25,26]$. The study's 35 -item screening tool includes questions from the Patient Health Questionnaire-9 (PHQ-9) [27] along with questions related to suicidal ideation and attempts, substance abuse, anger, stress, anxiety, eating disordered behavior, and current psychotherapy or psychopharmacological treatment. Participants are able to remain completely anonymous if they wish and identify themselves only with a user ID that they create. At the end of the survey, they are asked if they wish to provide an email address to allow for notification when the counselor has responded to their survey. Respondents who do not wish to provide an email address are instructed to $\log$ back into the site within $24 \mathrm{~h}$ and check for the counselor's response. Those who give an email address are sent a link to view the response.

The system's algorithm classifies participants into three different tiers depending on depression severity and suicide risk. High-risk respondents who have considered suicide during the past 2 weeks are classified as Tier 1A, while those experiencing severe distress during the past 4 weeks are classified as Tier 1B. Respondents experiencing mild to moderate distress are classified as Tier 2 and those experiencing no significant distress are classified as Tier 3.

\section{Screening and Assessment Phase}

Roughly once a year, residents and faculty were invited via email by their department chair to complete the Wellness Survey. In the last 2 years, the invitation is sent by the newly created office of the Chief Wellness Officer. The survey counselor provided feedback regarding risk level to all respondents through the system's messaging feature from a generic, tierspecific template. For Tier 1 and Tier 2, the template included 
emergency contact information and a list of therapists and psychiatrists. The online platform has a feature to show whether the counselor's message was opened by the intended recipient. The counselor sent one additional message to Tier 1 and Tier 2 respondents who did not open the first message after 1 week.

After receiving feedback from the counselor, Tier 1 and Tier 2 respondents were asked to choose among the following options: (1) do nothing further; (2) message anonymously with the counselor through the website; or (3) break their anonymity by speaking with the counselor in person, by phone or by email.

During the in-person assessment, the counselor reviewed the respondent's symptoms and history, as well as treatment options and appropriate referrals. Psychiatrist members of the WBC were available to the counselor for consultation.

\section{Follow-up Phase}

During the in-person assessment, respondents were asked to provide informed consent to participate in the follow-up portion of the study. Subsequently, they received an email every 3 months during 12 months from a specially created secure email account asking them to complete a 5-min follow-up questionnaire. Those who do not complete the follow-up questionnaires were emailed a total of three reminders.

\section{Results}

\section{Screening Data}

Results from data collected between February 4, 2013 and March 22, 2019, are included in this report, corresponding to five (approximately annual) waves of surveys. There were 3748 invitations sent to 1546 faculty physicians and 2202 resident physicians over the time covered by this report (Fig. 1).

Six hundred and thirty-nine surveys were returned complete, but 100 of them were excluded from the analyses for the following reasons: respondent identified as medical student $(n=40)$; multiple entries for the same username at different time points, in which case the most recent response was included in the analyses $(n=28)$; respondent identified as "other" $(n=21)$; preferred not to answer question of professional identity $(n=9)$; respondent identified as RN/NP $(n=1)$; and did not answer question on professional identity $(n=1)$. The remaining 539 valid surveys were used to conduct further analysis, 292 corresponding to faculty and 247 to residents (Fig. 1). Valid surveys corresponded to a response rate of $14.4 \%$.

Based on data from the 539 valid surveys, Table 1 shows that most respondents were classified at moderate (333 [62\%]) or high risk for suicide and depression (193 [36\%]), with only
$13(2 \%)$ classified as Tier 3. Ninety-one faculty members (31\%) and 102 residents or fellows (41\%) were classified as high risk for depression and/or suicide (Table 1).

Respondents at high or moderate risk for suicide and depression had mean PHQ-9 scores of 11.14 (SD 5.19) and 4.09 (SD 2.96), respectively. Ten (5\%) of the 193 Tier 1 respondents and $3(1 \%)$ of the 333 Tier 2 respondents reported past suicide attempts. Regarding treatment at the time of the survey,19 (10\%) of Tier 1 respondents reported psychotherapy, $25(13 \%)$ psychotropic medication, and $10(5 \%)$ both therapy and medication. Of the Tier 2 respondents, $19(6 \%)$ were receiving psychotherapy, 23 (7\%) were taking psychotropic medication, and $11(3 \%)$ were seeking both modes of treatment.

\section{Online Messaging and Referral Data}

Among the 526 Tier 1 and Tier 2 respondents, 212 (40\%) communicated with the counselor, including 103 who responded to the initial email with feedback from the counselor and 109 who answered the follow-up message. In order to determine the impact of our program on the referral of Tier 1 and Tier 2 respondents to mental health services, we first needed to exclude some of them for the following reasons. First, we excluded 10 respondents who bypassed the messaging system and contacted the counselor directly since we were interested in respondents' attitudes toward the survey's messaging feature. We then excluded 31 respondents because when answering the professional identity question they did not identify as resident, fellow, or faculty member (7), or identified as "other" (8), as medical student $(n=15)$, or preferred not to answer the question $(n=1)$. Last, we excluded 4 respondents because they were referred to non-mental health resources, such as Equal Opportunity Office, Office of Ombudsman, or their primary care provider.

After exclusions, 167 respondents who communicated with the counselor remained for consideration, 83 of whom received information regarding further evaluation and treatment and 84 who did not receive any study-based evaluation and referral. Figure 1 groups the 83 respondents who received treatment information according to the setting in which they received a treatment referral, in-person or online. The majority of these participants (48 or 58\%) were referred during an inperson encounter with the counselor. Table 1 shows that twothirds of the respondents referred to treatment (53 or 64\%) were classified as Tier 1 . The number of referrals for each of the five survey waves were $15,22,23,10$, and 13 .

\section{Outcomes from Follow-up Survey}

Fourteen respondents consented to the follow-up portion of the study, for the majority of whom the survey was the catalyst to seek an appointment with a mental health professional. 


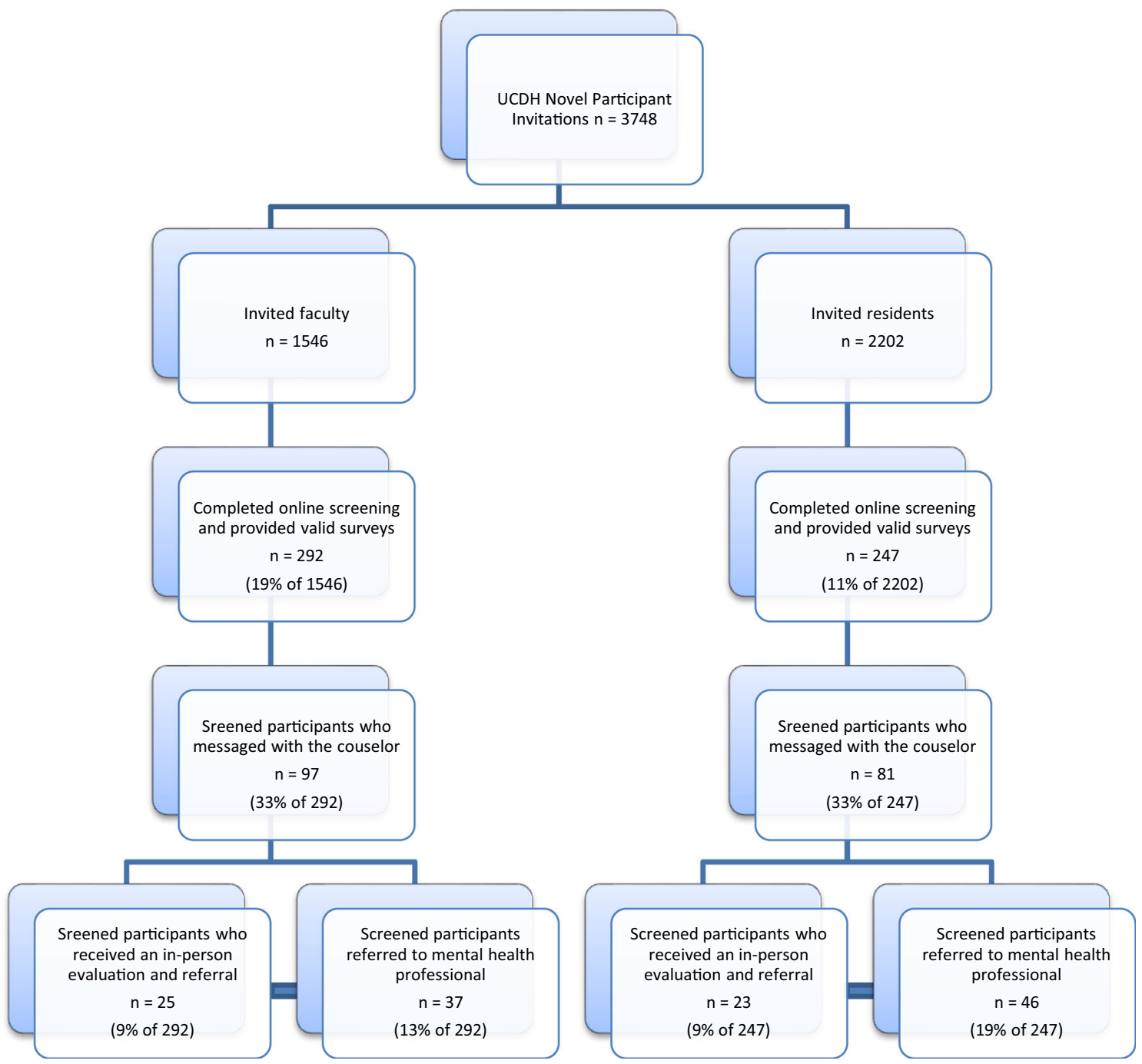

Fig. 1 New participants who were invited to take the survey, screened, assessed, and referred to UCDH Medical Staff Well-being Committee's Wellness Survey program from February 4, 2013 to March 22, 2019. Although there were 639 completed surveys, 100 responses were excluded from analysis either because they were identified as invalid as they did

not identify as a resident, fellow or faculty member (72), or there were multiple entries for the same username at different time points (28). In that case, only the most recent response was included in the analysis. The total number of valid surveys is 539

Specifically, six respondents indicated they would not have made an appointment to meet with a mental health professional without the survey and six had considered seeking treatment but had not acted on it until the survey. Only two respondents reported they would have sought an appointment with a mental health professional without the survey.

Seven of the fourteen respondents completed at least one follow-up survey (two returned the questionnaire at 3 months only; one returned the questionnaires at 3 and 6 months; one returned the questionnaire at 6 and 12 months; and three returned the questionnaire at all four follow-up surveys). These seven indicated that treatment was ongoing at followup. Six of the seven who completed the 3-month follow-up survey endorsed feeling improved after taking the survey. Among the four who completed the 12-month survey, one respondent endorsed feeling much improved, one improved,

and two feeling about the same. All four indicated they continued to engage in treatment, felt the survey was useful, and thought it should continue to be offered.

\section{Discussion}

As pointed out earlier, the barriers to help-seeking highlighted by this study are likely to continue to drive the COVID-19related mental health crisis among physicians. There is a critical need to escalate and improve initiatives by academic health centers and others to educate, enhance resilience, combat stigma, and increase accessibility of mental health services. In these pre-pandemic data, the percentage of physicians classified at moderate or high risk for depression and suicide that are referred to appropriate care each year has not 
Table 1 Results from the Wellness Survey and referrals by tier, UCDH, February 4, 2013-March 22nd, 2019

\begin{tabular}{|c|c|c|c|}
\hline Measure & $\begin{array}{l}\text { Tier } 1 \\
\text { (high risk) } \\
\text { No. }(\%)\end{array}$ & $\begin{array}{l}\text { Tier } 2 \\
\text { (moderate risk) } \\
\text { No. }(\%)\end{array}$ & $\begin{array}{l}\text { Tier } 3 \\
\text { (low risk) } \\
\text { No. }(\%)\end{array}$ \\
\hline Resident and fellows & $102(41.3 \%$ of 247$)$ & $144(58.3 \%$ of 247$)$ & $1(0.4 \%$ of 247$)$ \\
\hline Faculty & $91(31 \%$ of 292$)$ & $189(65 \%$ of 292$)$ & $12(4 \%$ of 292$)$ \\
\hline Total completed online screening tools & $193(36 \%$ of 539$)$ & $333(62 \%$ of 539$)$ & $13(2 \%$ of 539$)$ \\
\hline Past suicide attempt & $10(5 \%$ of 193$)$ & $3(1 \%$ of 333$)$ & 0 \\
\hline In treatment (psychotherapy) & $29(15 \%$ of 193$)$ & $30(9 \%$ of 333$)$ & $1(8 \%$ of 13$)$ \\
\hline In treatment (psychotropic medications) & $35(18 \%$ of 193$)$ & $34(10 \%$ of 333$)$ & 0 \\
\hline Mode of treatment (only psychotherapy) & $19(10 \%$ of 193$)$ & $19(6 \%$ of 333$)$ & $1(8 \%$ of 13$)$ \\
\hline Mode of treatment (only psychotropic medications) & $25(13 \%$ of 193$)$ & $23(7 \%$ of 333$)$ & 0 \\
\hline Mode of treatment-(both psychotherapy and psychotropic medications) & $10(5 \%$ of 193$)$ & $11(3 \%$ of 333$)$ & 0 \\
\hline In-person evaluation by counselor $(\%)$ & $36(19 \%$ of 193$)$ & $12(4 \%$ of 333$)$ & 0 \\
\hline Referred for further evaluation or treatment to mental health professional & $53(27 \%$ of 193$)$ & $30(9 \%$ of 333$)$ & 0 \\
\hline
\end{tabular}

increased over the years. In an institution with a physician turnover of about $14 \%$ residents and faculty per year $(250$ / $1800)$, the consistent rate of newly identified physicians at risk and referred to care is likely a reflection of the episodic nature of depression. This finding emphasizes the importance of performing regular universal screenings so as not to miss new episodes of illness. The UCDH Wellness Survey currently has ongoing funding within the institution and continues to email an average of 1800 surveys each year to residents and faculty. We expected that the efforts of the WBC and administrative leadership to educate UCDH physicians (e.g., regular grand rounds presentations) over the years of the study would result in increasing engagement with our program. However, the survey response rate has remained similar over the years. In the near future, we hope to see greater rates of response and engagement with the Wellness Survey as our institution ramps up efforts to address stigma toward mental health problems and help-seeking. As part of those efforts, the UCDH recently created the position of Chief Wellness Officer, which the senior author (PY) has filled.

Importantly, almost $60 \%$ of Tier 1 and Tier 2 respondents (314 out of 526) did not engage with the study counselor. We have no information as to whether those individuals eventually felt better with or without professional help or continued to struggle. This lack of program engagement is particularly worrisome in regards to the nearly 1 in 4 respondents classified as high risk ( $73 \%$ or 140 of 193). However, the small number of physicians who consented to participate in the follow-up portion of the program, reported treatment engagement, significant improvement over time, and encouraged us to continue this program.

Several limitations need to be acknowledged. First, both the unadjusted (17\%) and adjusted response rates (14\%) were low, although within the range of other non-compensated physician surveys [28, 29]. Structural stigma, defined as "societal-level conditions, cultural norms, and institutional policies that constrain the opportunities, resources, and wellbeing of the stigmatized" [30], likely caused many physicians to disregard the survey. The state of California is one whose medical licensing bodies requires physicians to answer questions regarding lifetime diagnosis and treatment of mental or substance abuse disorders. It is possible that we could do more to emphasize the confidential nature of the Wellness Survey to increase the response rate. To that end, we plan to have the Chief Wellness Officer send future surveys instead of relying on department chairs. Concerns about confidentiality may have also played a significant role when we consider that almost $60 \%$ of Tier 1 and Tier 2 respondents did not engage with the study counselor.

Although the staggering percentage of respondents were classified at risk $(98 \%)$ could suggest that our program is reaching out to physicians needing mental health treatment, we cannot assume that non-respondents are at low risk for depression and suicide. It is possible that a driver of nonresponse was the perception that the survey was not personally relevant; however, it is equally possible that non-response stemmed from depression itself (i.e., low motivation and hopelessness) or other concerns, such as confidentiality and confronting a stigmatizing diagnosis.

Avoidance of formal care by physicians are highlighted by findings from a multi-site longitudinal study of interns showing that only $22.7 \%$ who screened positive for depression reported receiving treatment [31]. Depressed interns who did not receive treatment were significantly more likely to prefer to manage their problems on their own and significantly less likely to believe that mental health treatment worked [31]. Also, in a convenience sample of 2106 female physicians representing all 50 states and the District of Columbia, almost 
$50 \%$ believed that they had met the criteria for mental illness but had not sought treatment [15]. The authors of this study speculate that the stigma associated with having a formal mental health diagnosis is a barrier to physicians seeking treatment [15].

The possibility that our survey captured a fraction of physicians struggling with depression and suicidal ideation is highlighted by a meta-analysis of international studies of depression in physician residents, which yielded a prevalence rate of depression of $20.9 \%$ using a comparable cutoff $\geq 10$ for the PHQ-9 [31] (the mean PHQ-9 of our Tier 1 respondents was 11). The 5-year results from a screening and referral program similar to ours, likewise found lower prevalence of depression, with rates of $6.6 \%, 10.2 \%$, and $8.4 \%$ among medical students, residents, and faculty, respectively [28].

A second limitation is that findings from this study pertain to a single academic medical center in the USA and cannot be generalized to other physicians in training or in practice, or in other countries. Third, information on depression and suicide was gathered with a self-report instrument. We are not aware of studies testing the validity of clinician-based versus selfreport diagnosis of depression among physicians.

As fourth limitation, we can point out that we did not collect demographic data such as age, gender, and race/ethnicity. This limits further our ability to test hypotheses (e.g., female physicians being more likely than male physicians to respond to the Wellness Survey and engage with the study counselor) or explore additional differences that could be tested in future studies (e.g., younger physicians being less likely than older physicians to be in treatment at the time of the survey).

Limitations notwithstanding, it is clear from the survey data that a significant minority of UCDH physicians were struggling psychologically or had denied or ignored their need to seek help for some time. The vast majority of physicians who responded to the Wellness Survey were at moderate or high risk. Notably, $72 \%$ of the physicians at high risk were not receiving any sort of treatment when surveyed (the corresponding percentage in the Moutier et al. study was 67\%) [24].

The act of asking physicians to share their emotional experiences was generally viewed by participants who consented to the follow-up study as a positive mental health intervention in and of itself. Notably, more than half of the message exchanges took place after the counselor reached out to a respondent, which underlines the importance of a personalized response to this type of survey.

In summary, we have confirmed that it is possible to successfully introduce an online, confidential screening, and referral program for depression and suicide risk in a large academic medical center, with the dual aims of engaging physicians in care and of promoting physician wellness throughout the institution. It is also noteworthy that there are consistent rates of participation and reported symptom severity over time, with very few repeat users. Of those who did provide follow-up data, it is encouraging to note that they seem to derive benefit from treatment.

Acknowledgements The authors' wish to thank Ann Haas, Ph.D., Senior Director of Education and Prevention with The American Foundation for Suicide Prevention, and Maggie Mortali, Director of The American Foundation for Suicide Prevention's Interactive Screening Program, for all their knowledge and support with this program. The authors' also wish to thank the University of California, Davis Health (UCDH) Physician Wellbeing Committee for its dedication in improving physician well-being, UCDH Administration and Human Resources for their financial support of this project, Department Chairs, and our physicians, residents and fellows who took the time to complete the Wellness Survey.

Funding This project was funded by the University of California, Davis Health.

\section{Declarations}

The study's protocol was reviewed and approved by the corresponding Institutional Review Board. Study participants provided informed consent approved by the IRB.

Ethical Considerations The University of California, Davis Health's Institutional Review Board approved this project. Approved 12/19/ 2012-07/18/2018; Protocol 358961. Approved 08/07/2018-03/08/2022; Protocol 1217375.

Conflict of interest On behalf of all authors, the corresponding author states that there is no conflict of interest.

\section{References}

1. Center C, Davis M, Detre T, Ford DE, Hansbrough W, Hendin H, et al. Confronting depression and suicide in physicians: a consensus statement. JAMA. 2003;289(23):3161-6.

2. Beckman $H$. The role of medical culture in the journey to resilience. Acad Med. 2015;90(6):710-2.

3. Thompson WT, Cupples ME, Sibbett CH, Skan DI, Bradley T. Challenge of culture, conscience, and contract to general practitioners' care of their own health: qualitative study. BMJ. 2001;323(7315):728-31.

4. Wallace JE, Lemaire JB, Ghali WA. Physician wellness: a missing quality indicator. Lancet. 2009;374(9702):1714-21.

5. Gross CP, Mead LA, Ford DE, Klag MJ. Physician, heal thyself? Regular source of care and use of preventive health services among physicians. Arch Intern Med. 2000;160(21):3209-14.

6. Berk J, Mills B, Varma S. Physician, heal thyself: health maintenance behaviors among physicians. Tex Med. 2014;110(12):e1.

7. Montgomery AJ, Bradley C, Rochfort A, Panagopoulou E. A review of self-medication in physicians and medical students. Occup Med. 2011;61(7):490-7.

8. Fox F, Harris M, Taylor G, Rodham K, Sutton J, Robinson B, et al. What happens when doctors are patients? Qualitative study of GPs. Br J Gen Pract. 2009;59(568):811-8.

9. Henderson M, Brooks SK, del Busso L, et al. Shame! Selfstigmatisation as an obstacle to sick doctors returning to work: a qualitative study. BMJ Open. 2012;2(5):1-8. 
10. Brimstone R, Thistlethwaite JE, Quirk F. Behaviour of medical students in seeking mental and physical health care: exploration and comparison with psychology students. Med Educ. 2007;41(1):74-83.

11. Hooper C, Meakin R, Jones M. Where students go when they are ill: how medical students access health care. Med Educ. 2005;39(6): 588-93.

12. Tyssen R, Røvik JO, Vaglum P, Grønvold NT, Ekeberg Ø. Helpseeking for mental health problems among young physicians: is it the most ill that seeks help? Soc Psychiatry Psychiatr Epidemiol. 2004;39(12):989-93.

13. Roberts LW, Warner TD, Trumpower D. Medical students' evolving perspectives on their personal health care: clinical and educational implications of a longitudinal study. Compr Psychiatry. 2000;41(4):303-14.

14. Hill AB. Breaking the stigma - a physician's perspective on selfcare and recovery. N Engl J Med. 2017;376(12):1103-5.

15. Gold KJ, Andrew LB, Goldman EB, Schwenk TL. "I would never want to have a mental health diagnosis on my record": a survey of female physicians on mental health diagnosis, treatment, and reporting. Gen Hosp Psychiatry. 2016;43:51-7.

16. Rodriguez RM, Medak AJ, Baumann BM, Lim S, Chinnock B, Frazier R, et al. Academic emergency medicine physicians' anxiety levels, stressors, and potential stress mitigation measures during the acceleration phase of the COVID-19 pandemic. Acad Emerg Med. 2020;27(8):700-7.

17. Al Sulais E, Mosli M, AlAmeel T. The psychological impact of COVID-19 pandemic on physicians in Saudi Arabia: a crosssectional study. Saudi J Gastroenterol. 2020;26(5):249-55.

18. Luceño-Moreno L, Talavera-Velasco B, García-Albuerne Y, Martín-García J. Symptoms of posttraumatic stress, anxiety, depression, levels of resilience and burnout in Spanish health personnel during the COVID-19 pandemic. Int J Environ Res Public Health. 2020;17(15):5514.

19. Kannampallil TG, Goss CW, Evanoff BA, Strickland JR, McAlister RP, Duncan J. Exposure to COVID-19 patients increases physician trainee stress and burnout. PLoS One. 2020;15(8):e0237301.

20. Matsuo T, Kobayashi D, Taki F, Sakamoto F, Uehara Y, Mori N, et al. Prevalence of health care worker burnout during the coronavirus disease 2019 (COVID-19) pandemic in Japan. JAMA Netw Open. 2020;3(8):e2017271.
21. Duarte D, El-Hagrassy MM, TCE C, Gurgel W, Fregni F, Correa H. Male and female physician suicidality: a systematic review and meta-analysis. JAMA Psychiatry. 2020;77(6):587-97.

22. Yaghmour NA, Brigham TP, Richter T, Miller RS, Philibert I, Baldwin DWC Jr, et al. Causes of death of residents in ACGMEaccredited programs 2000 through 2014: implications for the learning environment. Acad Med. 2017;92(7):976-83.

23. Haskins J, Carson JG, Chang CH, Kirshnit C, Link DP, Navarra L, et al. The suicide prevention, depression awareness, and clinical engagement program for faculty and residents at the University of California, Davis Health System. Acad Psychiatry. 2016;40(1):239.

24. Moutier C, Norcross W, Jong P, Norman M, Kirby B, McGuire T, et al. The suicide prevention and depression awareness program at the University of California, San Diego School of Medicine. Acad Med. 2012;87(3):320-6.

25. Moffitt LB, Garcia-Williams A, Berg JP, Calderon ME, Haas AP, Kaslow NJ. Reaching graduate students at risk for suicidal behavior through the interactive screening program. J Coll Stud Psychother. 2014;28(1):23-34.

26. Haas A, Koestner B, Rosenberg J, Moore D, Garlow SJ, Sedway J, et al. An interactive web-based method of outreach to college students at risk for suicide. J Am Coll Heal. 2008;57(1):15-22.

27. Spitzer RL, Kroenke K, Williams JW, and the Patient Health Questionnaire Primary Care Study G. Validation and utility of a self-report version of prime-md: the phq primary care study. JAMA. 1999;282(18):1737-44.

28. Zisook S, Young I, Doran N, Downs N, Hadley A, Kirby B, et al. Suicidal ideation among students and physicians at a U.S. medical school: a healer education, assessment and referral (HEAR) program report. Omega (Westport): Journal of Death and Dying. 2016;74(1):35-61.

29. Cook DA, Wittich CM, Daniels WL, West CP, Harris AM, Beebe TJ. Incentive and reminder strategies to improve response rate for internet-based physician surveys: a randomized experiment. J Med Internet Res. 2016;18(9):e244.

30. Hatzenbuehler ML. Structural stigma: research evidence and implications for psychological science. Am Psychol. 2016;71(8):74251.

31. Guille C, Speller H, Laff R, Epperson CN, Sen S. Utilization and barriers to mental health services among depressed medical interns: a prospective multisite study. J Grad Med Educ. 2010;2(2):210-4.

Publisher's Note Springer Nature remains neutral with regard to jurisdictional claims in published maps and institutional affiliations. 